\title{
Accuracy of visible retinal emboli for the detection of cardioembolic lesions requiring anticoagulation or cardiac surgery
}

\author{
Sanjay Sharma, Gary C Brown, Alan F Cruess for the Retinal Emboli of Cardiac Origin \\ study group
}

\begin{abstract}
Aim-To determine the accuracy of visible retinal emboli as a diagnostic "test" for the likelihood of receiving anticoagulation or cardiac surgery based on the results of transthoracic echocardiography, in the setting of acute retinal arterial occlusion. Methods-A multicentre retrospective diagnostic study at Kingston Eye Centre, Queen's University, Kingston, Ontario; Wills Eye Hospital, Philadelphia; Ottawa Eye Institute, Ottawa, Ontario; and the Halifax Infirmary, Halifax, Nova Scotia of 104 patients with both embolic and nonembolic acute retinal arterial obstruction who underwent transthoracic echocardiography was performed, to determine the accuracy of visible retinal emboli as a diagnostic "test" for anticoagulation or cardiac surgery. Anticoagulation or surgical intervention on the basis of abnormalities was detected solely through the technology of transthoracic echocardiography.

Results-41 patients had visible retinal emboli (calcific, cholesterol, or fibrin). The remaining 63 had no evidence of embolic disease. The sensitivity of emboli for the likelihood of a patient receiving anticoagulation or cardiac surgery was $50 \%$. The specificity, positive predictive value, and negative predictive value were $62 \%, 15 \%$, and $90 \%$, respectively. The likelihood ratio ( $L R=1.31)$ obtained given the presence of a visible retinal embolus was neither clinically nor statistically significant $(\mathrm{LR}+\mathrm{ve}=1.31 ; 95 \%$ CI $(0.91,3.16))$. This likelihood ratio, when applied to a patient with a pretest probability of $50 \%$, results in a post-test probability of $56.7 \%$. Conclusions-These results demonstrate that the presence of a visible retinal embolus should not be the sole determinant of whether to order transthoracic echocardiography, as the likelihood ratio for a patient receiving anticoagulation or cardiac surgery, given the presence of a visible retinal embolus was only 1.31 . (Br f Ophthalmol 1998;82:655-658)
\end{abstract}

Patients with acute retinal arterial obstruction commonly present with sudden visual loss. Embolic retinal arterial obstruction is associated with a higher risk in overall mortality when compared with non-embolic occlusion. ${ }^{1}$ Retinal emboli may be of cardiac origin, repre- sent material derived from carotid artery stenosis, or may represent platelet fibrin aggregation. $^{2}$

The following cardiac pathology has been reported in cases of acute retinal arterial obstruction: aortic and mitral valve disease, ${ }^{3-5}$ atrial myxomas, ${ }^{6}$ valvulotomy, ${ }^{7}$ angioplasty, ${ }^{8}$ acute myocardial infarction, ${ }^{9}$ subacute bacterial endocarditis, intravenous drug related talc embolisation, ${ }^{10}$ prosthetic valves, ${ }^{11}$ and mitral valve prolapse..$^{12}$ The prevalence of cardiac valvular disease in cases presenting with acute retinal arterial obstruction has been reported to be approximately $25 \% .{ }^{36}$ Accordingly, it has been recommended that transthoracic echocardiography be performed in the evaluation of acute retinal arterial obstruction, especially in the presence of visible retinal emboli. $^{2}{ }^{4}$

By modifying the probability of disease, historical facts, and physical findings act in a fashion similar to a diagnostic test. ${ }^{15}{ }^{15}$ In fact, the accuracy of physical findings can be quantitated in terms of sensitivity, specificity, and predictive values. In this report we determine if the presence of a visible retinal embolus increases the probability of an acute retinal arterial occlusion patient receiving anticoagulation or cardiac surgery.

\section{Patients and methods}

The charts of 243 patients with acute retinal arterial obstruction were consecutively reviewed. The charts were obtained from four tertiary, North American hospital centres. From the initial 243, 104 met the following inclusion criteria:

(i) Acute central retinal or branch retinal arterial obstruction on the basis of clinical examination (either clinical cloudy swelling or an arterial dye front on angiography).

(ii) Clear documentation of the presence of retinal emboli (patients' charts or fundus photographs). We documented the presence or absence of emboli. Qualitative assessment regarding the nature of the emboli was not undertaken, because of its subjective nature. ${ }^{16}$

(iii) A clinical cardiac evaluation on the basis of past history for relevant cardioembolic high risk factors including presence of any of the following: subacute bacterial endocarditis, rheumatic heart disease, mitral valve prolapse, recent myocardial infarction, a prosthetic valve, cardiac tumour, intravenous drug use, congenital heart
Accepted for publication 20 January 1998 
Table 1 Anticoagulation or cardiac surgery in acute retinal arterial occlusion patients, given embolic status

\begin{tabular}{lclc}
\hline & $\begin{array}{l}\text { Anticoagulation or cardiac } \\
\text { surgery }\end{array}$ & $\begin{array}{l}\text { No anticoagulation or } \\
\text { cardiac surgery }\end{array}$ & Totals \\
\hline Visible retinal embolus & 6 & 35 & 41 \\
No embolus & 6 & 57 & 63 \\
Totals & 12 & 92 & 104 \\
\hline
\end{tabular}

disease, or valvular heart disease, the presence of a murmur detected at presentation and any electrocardiographic risk factors for embolisation (atrial fibrillation, acute ST elevation or Q-waves).

(iv) Transthoracic echocardiographic evaluation.

(v) The decision to anticoagulate (heparin or coumadin) or to surgically intervene based on an echocardiographic result was recorded.

The patients were then divided into one of two groups, based on the presence or the absence of visible retinal emboli. These two groups were then analysed with respect to whether they received anticoagulation or cardiac surgical intervention based on their echocardiographic result.

The study was designed to detect a likelihood ratio (positive test) of 4 , given the presence of a visible retinal embolus. This likelihood ratio was deemed clinically significant, as it is the value at which a pretest probability of $50 \%$ translates to a post-test probability of $80 \%$. The study was designed to detect this difference with a power of $80 \%$ (type II error = 0.20 ) and a type I error of 0.05 . We summarised our data by way of a contingency table. Likelihood ratios were calculated as described by Sackett et al. ${ }^{15}$ Ninety five per cent confidence intervals ${ }^{17}$ were calculated around likelihood ratios obtained in both the presence and absence of retinal emboli, to assess their statistical significance.

\section{Results}

The mean age of our population was 60.9 years. Forty one $(39 \%)$ of the patients had visible retinal emboli, and the remaining $63 \mathrm{had}$ no evidence of visible retinal emboli.

Forty eight echocardiograms were reported as abnormal. From these, however, only 12 patients $(11 \%)$ received anticoagulation or cardiac surgery (Table 1). Of these 12 patients, six had visible retinal emboli. Thus, the sensitivity of visible retinal emboli for the likelihood of a patient requiring anticoagulation or cardiac surgery was $50 \%$ (Table 2 ). The specificity was $61.9 \%$.

Table 2 Test variables regarding visible retinal for the outcome of anticoagulation or cardiac surgery

\begin{tabular}{ll}
\hline Diagnostic test variable & $\begin{array}{l}\text { Visible retinal } \\
\text { embolus }\end{array}$ \\
\hline Sensitivity & $50 \%$ \\
Specificity & $62 \%$ \\
Positive predictive value & $14.6 \%$ \\
Negative predictive value & $90.5 \%$ \\
Likelihood ratio given a positive test & 1.31 \\
Likelihood ratio given a negative test & 0.807 \\
\hline
\end{tabular}

EMBOLIC GROUP

Forty one patients had visible retinal emboli. Six of 41 embolic patients (15\%) required anticoagulation or cardiac surgery based on their echocardiograms. The remaining 35 (85 $\%)$, required no intervention. The six embolic patients who were anticoagulated or had cardiac surgery had the following cardiac pathology detected-mitral valve prolapse (one case), atrial myxoma (one case), aortic stenosis (one case), a cardiac mural thrombus (two cases), and an acute myocardial infarction (one case). The positive predictive value of the presence of a visible retinal embolus for anticoagulation or cardiac surgery was $14.6 \%$ (Table 2 ).

\section{NON-EMBOLIC GROUP}

Sixty three patients with acute retinal arterial obstruction did not have visible retinal emboli. Of these patients, six ( $8 \%$ ) received anticoagulation or cardiac surgery based on their echocardiographic results (Table 1). The following pathologies required anticoagulation in the non-embolic group - vegetations on aortic valve (one case), aortic stenosis (two cases), and mitral valve stenosis (three cases). The negative predictive value of the absence of a visible retinal embolus was $90 \%$.

The likelihood ratio for anticoagulation or cardiac surgery, given the presence of visible retinal embolus was $1.31 ; 95 \%$ CI $(0.91,3.16)$. The likelihood ratio for anticoagulation or cardiac surgery, given the absence of a visible retinal embolus, was $0.807 ; 95 \%$ CI $(0.447,1.45)$.

\section{Discussion}

Acute retinal arterial obstruction has been associated with cardiac valvular disease in approximately $25 \%$ of cases. ${ }^{3}{ }^{4}$ Visible retinal emboli are a clinical sign that a retinal arterial obstruction may be associated with carotid or cardiac pathology. ${ }^{18}{ }^{19}$ Accordingly, it has been recommended that acute retinal arterial obstruction in the setting of visible emboli be aggressively investigated to rule out a potential cardiac source for the emboli. ${ }^{2}$ These recommendations have been made on the basis of prevalence figures obtained by historical data, studies with few numbers, or based on deductive reasoning to search for a proximal embolic source.

Our data reveal that $46 \%$ (48 of 104) of our patients had detectable abnormalities on their echocardiograms. Owing to the fact that there are numerous abnormalities detected on echocardiography for which no intervention is required, the more important outcome measure is anticoagulation or cardiac surgery, based on these abnormalities. In our series, only 12 of 104 patients $(11 \%)$ with acute retinal arterial obstruction had findings on their echocardiograms which required anticoagulation or surgical intervention (Table 1). Six of the 12 had visible retinal emboli, whereas the remaining six presented with an acute retinal arterial obstruction which lacked any embolic material. With a sensitivity of $50 \%$, half of the patients with cardiac pathology requiring anticoagulation or cardiac surgery would have 
Table 3 Probability of anticoagulation or cardiac surgery given varying pretest probabilities

\begin{tabular}{lll}
\hline $\begin{array}{l}\text { Patients with hypothetical } \\
\text { pretest probabilities }\end{array}$ & $\begin{array}{l}\text { Post-test probability given the } \\
\text { presence of a visible retinal embolus }\end{array}$ & $\begin{array}{l}\text { Post-test probability given } \\
\text { absence of retinal embolus }\end{array}$ \\
\hline Patient A: $10 \%$ & $12.7 \%(+2.7)$ & $8.2 \%(-1.8)$ \\
Patient B: $50 \%$ & $56.7 \%(+6.7)$ & $44.6 \%(-5.4)$ \\
Patient C: $90 \%$ & $92.1 \%(+2.1)$ & $87.9 \%(-2.1)$ \\
\hline
\end{tabular}

been missed if one had not ordered echocardiography in the absence of visible retinal emboli.

Our results reveal that the likelihood for anticoagulation or cardiac surgery was $1.31 \times$ higher in the presence of a visible retinal embolus $(\mathrm{LR}+\mathrm{ve}=1.31)$. This likelihood ratio was not clinically significant, as it would result in a post-test probability of $56.7 \%$, given a patient with a pretest probability of $50 \%$-an increase in the probability of only $6.7 \%$ (Table 3 ). The value obtained for our positive likelihood ratio was not statistically significant, as its $95 \%$ CI $(0.91,3.16)$ includes 1 . Given that our study was designed to detect a likelihood ratio of 4 , and that the upper limit of our $95 \%$ CI was 3.16 , we are able to reject our alternate hypothesis.

The likelihood of a patient receiving anticoagulation or cardiac surgery was $0.807 \times$ lower in the absence of a visible retinal embolus $(\mathrm{LR}-\mathrm{ve}=0.807)$. This likelihood ratio, too, is not clinically significant, as the post-test probability for anticoagulation or cardiac surgery decreases to $45 \%$, given a pretest probability of $50 \%$ - a decrease of only $5 \%$ (appendix 2). The value obtained for the likelihood ratio obtained in the absence of an embolus is not statistically significant, as the $95 \%$ CI $(0.447$, 1.45) includes 1 .

Overall, the presence or absence of a visible retinal embolus, in the setting of acute retinal arterial occlusion, did not significantly alter the probability of anticoagulation or cardiac surgery. This is true for hypothetical patients with varying pretest probabilities (Table 3 ).

A recent study by our group showed the importance of a careful clinical assessment which included a full functional inquiry and a cardiac auscultatory examination. ${ }^{20}$ That study demonstrated that, if one or more cardioembolic risk factor was present, a patient was 25 times more likely to require anticoagulation or cardiac surgery than if no risk factors was present $(\mathrm{OR}=25,95 \% \mathrm{CI}(3.04-217.02)){ }^{20}$

In any cross sectional study, there is always the possibility of misclassification of patients with respect to different variables. As this cross sectional study was retrospective in nature, non-differential misclassification may have been introduced. With respect to our "diagnostic" test, that being the presence or absence of emboli, it was unlikely that misclassification of visible emboli occurred, as most clinical assessments were confirmed with fundus photography. However, in the absence of visible emboli, patients were classified as non-embolic. Nonembolic cases could have, in fact, been of embolic pathogenesis, with the emboli disintegrating before ophthalmoscopy. With respect to the outcome variable of anticoagulation or cardiac surgical intervention, there also was little opportunity to misclassify patients. However, owing to the retrospective nature of the study, the variable of anticoagulation or cardiac surgery actually represents management that was recommended based on the echocardiographic results. These recommendations were made by different cardiologists, based on their own criteria. Certain cardiac pathologies may be handled differently, but without the adoption of various evidence based consensus statements of how to manage different echocardiographic abnormalities, it is difficult to evaluate whether misclassification of our outcome may have occurred.

It should also be noted that the outcome variable of our study (anticoagulation or cardiac surgery) was based on the results of transthoracic echocardiography, as opposed to transoesophageal data. However, there is evidence that certain cardiac pathology detected solely with transoesophageal echocardiography may be associated with acute retinal arterial obstruction. ${ }^{21}$ Given the low prevalence of acute retinal arterial obstruction and the relatively recent use of the technique of transoesophageal echocardiography, a prospective study would have to be undertaken to evaluate this hypothesis.

In summary, the presence of a visible retinal embolus in the setting of acute retinal arterial obstruction did not clinically or statistically increase the likelihood of a patient receiving anticoagulation or cardiac surgery, based on transthoracic echocardiography.

\section{Appendix 1. Calculation of post-test probability, in the presence of a visible retinal embolus}

post-test probability $=$ post-test odds $/ 1+$ posttest odds

The post-test odds $=$ pretest odds $\times$ LR - ve, where the pretest odds $=$ pretest probability/1 - pretest probability

Example: assuming a hypothetical acute retinal arterial occlusion patient has 50:50 chance of having a cardiac lesion which required anticoagulation or cardiac surgery. How much would their probability change if we were to detect an embolus on ophthalmoscopy?

pretest odds $=0.5 / 1-0.5=1.0$

post-test odds $=($ pretest odds $)(L R+v e$ test $)$. Given that our data reveal that the LR+ve test $=1.31$, the post-test odds $=1.31$. Accordingly, the post-test probability is equal to $1.31 / 2.31$ or $57 \%$. Thus, in the presence of a visible retinal embolus, we have gone from a situation where the probability of HSCAS was $50 \%$, to one where it is $57 \%$.

\section{Appendix 2. Calculation of post-test probability, in the absence of a visible retinal embolus}

Example: Again, assuming that a patient with acute retinal arterial occlusion who has a 50:50 chance of having cardiac pathology requiring intervention, how much does their probability change if an embolus is not present on ophthalmoscopy? 
pretest odds $=0.5 / 1-0.5=1$

post-test odds $=$ pretest odds $\times$ LR-ve. Given our data reveals that LR-ve $=0.807$, post-test odds $=(1)(0.807)=0.807$. Accordingly, the post-test probability is equal to $0.807 / 1.807$ or 0.446 . Thus, we have gone from a situation where the probability of HSCAS was $50 \%$ to one where it is now $44.6 \%$.

The RECO (Retinal Emboli of Cardiac Origin) study group consists of the following members: Principal investigator: Sanjay

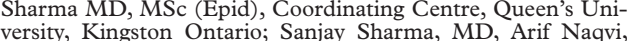
versity, Kingston Ontario; Sanjay Sharma, MD, Arif Naqvi,
MD, Susan M Sharma, MD, and Alan F Cruess MD; $\mathrm{MD}$, Susan $M$ Sharma, $\mathrm{MD}$, and Alan F Cruess MD;
co-investigators: Gary C Brown, MD, Wills Eye Hospital; Nils Mungan, MD, and Bruce Jackson, MD, The Ottawa Eye Institute; Ann Hoskin Mott, MD, The Nova Scotia Eye Centre.

1 Savino PJ, Glaser JS, Cassady J. Retinal stroke: is the patient at risk? Arch Ophthalmol 1977;95:1185-9.

2 Graham EM. The investigation of patients with retinal vascular occlusion. Eye 1990;4:464-8.

3 Wilson LA, Warlow CP, Russel RWR. Cardiovascular disease in patients with retinal arterial occlusion. Lancet 1979;10:292-4.

4 Appen RE, Wray SH, Cogan DG. Central retinal artery occlusion. Am f Ophthalmol 1975;79:374-81.

5 Brown GC, Magargal LE. Central retinal artery obstruction and visual acuity. Ophthalmology 1982;89:14-19.

6 Sybers HD, Boake WC. Coronary and retinal embolism from a left atrial myxoma. Arch Pathol 1971;91:179-82.

7 Holley KE, Bahn RC, McGoon DC, et al. Calcific embolization associated with valvotomy for calcific aortic stenosis. Circulation 1963;27:175-81.
8 Bucci FA, Dimitsopulos TM, Krohel GB. Branch retinal artery occlusion secondary to percutaneous transluminal coronary angioplasty. Br f Ophthalmol 1989;73:309-10.

9 Zimmerman LE. Embolism of the central retinal arterysecondary to myocardial infarction with mural thrombus. Arch Ophthalmol 1965;73:822-6.

10 Kresca LJ, Goldberg MF, Jampol LM. Talc emboli and retinal neovascularization in a drug user. Am f Ophthalmol 1979;87:334-9.

11 Ide $\mathrm{CH}$, Almond $\mathrm{CH}$, Hart WM, et al. Hematogenous dissemination of microemboli. Eye findings in a patient with Starr-Edwards aortic prosthesis. Arch Ophthalmol 1971;85: 614-17.

12 Wilson LA, Keeling PWN, Malcolm AD, et al Visual complications of mitral leaflet prolapse. BMf 1977;9:86-8.

13 Woldoff HS, Gerber M, Desser KB, et al. Retinal vascular lesions in two patients with prolapsed mitral valve leaflets. Am f Ophthalmol 1975;79:382-5.

4 Sackett DL, Rennie D. The science of the art of the clinical examination. $7 A M A$ 1992;267:2650-2.

15 Sackett DL, Haynes RB, Guyatt GH, et al. Clinical epidemiology: a basic science for clinical medicine. 2nd ed. Boston: Little, Brown, 1991:119-39.

16 Sharma S, Cruess AF, Pater J, et al. Can different types of retinal emboli be reliably differentiated from one another? Can 7 Ophthalmol (in press).

17 Simel DL, Samsa GP, Matchar DB. Likelihood ratios with confidence: sample size estimation for diagnostic test studies. F Clin Epidemiol 1991;44:763-70.

18 O'Donnell BA, Mitchell P. The clinical features and associations of retinal emboli. Aust f Ophthalmol 1992;20:11-7.

19 Younge BR. The significance of retinal emboli. I Clin Neuro-Ophthalmol 1989;9:190-4.

20 Sharma S, Naqvi A, Sharma SM, et al. Transthoracic echocardiographic findings in patients with acute retinal arterial obstruction: a retrospective review. Arch Ophthalmol 1996;114:1189-92.

21 Wiznia RA, Pearson WN. Use of transesophageal echocardiography for detection of a likely source of embolization to the central retinal artery. Am f Ophthalmol 1991;111:104-5. 